\title{
The Nijmegen high field magnet laboratory and its experimental facilities
}

\author{
J.A.A.J. Perenboom*, K. van Hulst, S.A.J. Wiegers, J.C. Maan \\ High Field Magnet Laboratory and Research Institute for Materials, University of Nijmegen, NL-6525 ED Nijmegen, The Netherlands
}

\begin{abstract}
The High Field Magnet Laboratory at the University of Nijmegen is capable of producing continuous magnetic fields up to $30 \mathrm{~T}$. The highest fields are generated using hybrid magnet systems which combine an external superconducting magnet providing the background field with a smaller resistive coil energised from a $6 \mathrm{MW}$ power supply.

For effective use of these facilities a broad range of instrumentation has been developed. Of great importance is the capability to maintain millikelvin temperatures, and a plastic dilution refrigerator designed and constructed at Nijmegen has demonstrated a base temperature of well below $30 \mathrm{mK}$ in $30 \mathrm{~T}$.
\end{abstract}

\section{Introduction}

The High Field Magnet Laboratory at the University of Nijmegen provides static magnetic fields up to $30 \mathrm{~T}$, together with a wide range of experimental facilities and expertise. The characteristic feature is that the magnetic field strength can in principle be maintained as long as the experiment requires, so that all experiments can be performed, more or less independent of their degree of sophistication: there is sufficient time for data acquisition to determine high-resolution spectra, to improve signal to noise ratios through averaging of weak but repeated signals, to allow for long (e.g. thermal) relaxation times, or to maintain temperatures in the millikelvin range. These magnetic fields and the additional facilities are available in a user-friendly way for investigations concerning fundamental physics and materials research.

A power supply of $6 \mathrm{MW}$ and a closed-cycle water cooling system allow to operate a number of resistive magnets, and two "hybrid" magnet systems. The hybrid

\footnotetext{
* Corresponding author.
}

magnets consist of an external superconducting magnet providing the background field and a smaller resistive coil at its centre and thus produce the highest possible continuous fields. The high fields are available on workdays from 19:00 hours and in the weekends; during normal working hours operation is also possible but restrictions apply. The installation has been described in several earlier publications [1-3], and a list of the presently installed magnets is given in Table 1. A few lower field purely superconductive magnets have been added to the list for completeness. These magnets are very useful for the preparation of the experiments, allowing a more efficient use of the scarce magnet time at the higher field strengths.

\section{Research in high magnetic fields}

In the past, a range of tools and techniques has been developed at the Nijmegen High Field Magnet Laboratory for investigations up to the highest magnetic fields. These additional facilities may also be made available to guest researchers for collaborative research projects. 
Table 1

High field magnets at the Nijmegen High Field Magnet Laboratory

\begin{tabular}{|c|c|c|c|c|}
\hline Magnet system & $\begin{array}{l}B_{\max } \\
(\mathrm{T})\end{array}$ & $\begin{array}{l}\text { Bore } \\
(\mathrm{mm})\end{array}$ & $\begin{array}{l}\text { Homogeneity } \\
\text { (1 cm dsv) }\end{array}$ & $\begin{array}{l}\text { Maximum power } \\
\text { (MW) }\end{array}$ \\
\hline 1. Hybrid magnet N-I & 25.0 & 53 & $3 \times 10^{-3}$ & 6.00 \\
\hline 2. Duplex Bitter coil & 20.0 & 32 & $1 \times 10^{-3}$ & 5.80 \\
\hline 3. Bitter coil & 15.2 & 60 & $2 \times 10^{-4}$ & 5.85 \\
\hline 4. Duplex Bitter coil & 20.0 & 32 & $1 \times 10^{-3}$ & 5.80 \\
\hline 5. Hybrid magnet N-II & 30.4 & 32 & $3 \times 10^{-3}$ & 5.50 \\
\hline 6. Superconducting coil & 17.5 & 32 & $1 \times 10^{-3}$ & $\mathrm{~N} / \mathrm{A}$ \\
\hline 7. Superconducting coil & 14.0 & 40 & $1 \times 10^{-3}$ & $\mathrm{~N} / \mathrm{A}$ \\
\hline
\end{tabular}

The spectroscopic tools span the whole range from microwaves, through the infrared to the visible, and include the following:

-Photoluminescence and photoluminescence-excitation spectroscopy, reflection, transmission and absorption in the visible ( $\lambda$ between 500 and $1000 \mathrm{~nm}$ ), in magnetic fields up to $25 \mathrm{~T}$ and at temperatures between $300 \mathrm{mK}$ and $300 \mathrm{~K}$; time-resolved spectroscopy in the $100 \mathrm{fs}$ and 3 ps domain; polarisation-dependent studies are possible; Faraday rotation.

- Raman spectrometer in combination with the $30 \mathrm{~T}$ hybrid magnet and at low temperatures.

-Far-infrared spectroscopy using far-infrared lasers in the range of wavelengths from 25 to $1500 \mu \mathrm{m}$; transmission, reflection and absorption in magnetic fields to $30 \mathrm{~T}$ and at temperatures down to $300 \mathrm{mK}$.

-Far-infrared Fourier transform spectroscopy at wave numbers from 1.2 to $10000 \mathrm{~cm}^{-1}$ using a slow-scan or rapid-scan Fourier spectrometer in magnetic fields up to $30 \mathrm{~T}$ and at temperatures down to $300 \mathrm{mK}$.

-Microwave spectroscopy in the frequency range 9-220 GHz using a Millimeter Wave Vector Analyzer in magnetic fields up to $30 \mathrm{~T}$.

The cryogenic facilities include simple bath cryostats and variable-temperature cryostats for the temperature range $1.2-300 \mathrm{~K}$. For the subkelvin range there are ${ }^{3} \mathrm{He}$ cryostats, and temperatures down to $25 \mathrm{mK}$ can be realised with plastic ${ }^{3} \mathrm{He} /{ }^{4} \mathrm{He}$ dilution refrigerators for magneto-transport and thermodynamic measurements in magnetic fields up to $30 \mathrm{~T}$.

Liquid cells are available to apply hydrostatic pressures to $25 \mathrm{kbar}$, and can be used up to $30 \mathrm{~T}$ for magnetotransport and up to $25 \mathrm{~T}$ for far-infrared measurements.

More specific set-ups for thermodynamic and magneto-transport experiments, which have been developed in the past and are available for collaborative research projects, include the following:
-Magnetostriction and thermal expansion with $0.05 \mathrm{~nm}$ resolution in magnetic fields up to $25 \mathrm{~T}$ and at temperatures between 1.2 and $20 \mathrm{~K}$.

Specific heat in magnetic fields up to $25 \mathrm{~T}$ and at temperatures between 1.2 and $20 \mathrm{~K}$.

Thermopower and Nernst-Ettinghausen effect at temperatures down to $50 \mathrm{mK}$ and in magnetic fields up to $30 \mathrm{~T}$.

- Magnetisation measurements using the ballistic extraction technique (sensitivity $10^{-6} \mathrm{~J} / \mathrm{T}$ ) in magnetic fields up to $20 \mathrm{~T}$ and at temperatures between 1.2 and $330 \mathrm{~K}$. -Torsion magnetometer with minimum detectable torsion moment $5 \times 10^{-11} \mathrm{Nm}$ in magnetic fields up to $30 \mathrm{~T}$ and at $1.2 \mathrm{~K}$.

-Magneto-transport measurements: resistance, viscosity, and furthermore capacitance, mutual inductance, etc.

-Critical-current measurements of superconducting wires, and its dependence on magnetic field $B$, temperature $T$ and strain $\varepsilon$.

-Magnetic separation; visual observation in situ using videocamera in $20 \mathrm{~T}$.

The laboratory not only provides service to outside users by making equipment and magnetic fields available to them, but also pursues a strong in-house research programme. This activity is essential in order to be able to retain affinity with active research areas, and to develop and maintain state-of-the-art equipment. Much of the equipment listed above was developed by the scientists at the laboratory for their own research, and can now be made available more widely.

The present research interests of the scientists at the Nijmegen High Field Magnet Laboratory include semiconductor physics, especially materials and lowdimensional devices, superconductivity, especially high- $T_{\mathrm{c}}$ superconductors and high-field conductors for technological applications, organic conductors, especially quasi-2D BEDT-TTF charge transfer salts and quasi-1D Bechgaard salts, intermetallic compounds, especially heavy 
fermion compounds, and quantum liquids, especially highly polarised dilute mixtures of ${ }^{3} \mathrm{He}$.

\section{High magnetic fields and low temperatures: a plastic dilution refrigerator}

A high magnetic field is a very general tool to study the electronic properties of materials. With available field strengths, its influence is only weak compared to the Coulomb energy, etc. The magnetic field can however induce new physical states manifesting phenomena such as the fractional quantum hall effect and field induced Wigner solid in the high mobility 2D electron gas, fieldinduced spin density waves in quasi-1D conductors, or quenching of spin fluctuations in itinerant ferromagnets and heavy fermion systems.

In all these cases maintaining very low temperatures is also imperative, and in many cases the determining parameter is in fact the ratio $B / T$. Although it is quite feasible to design and build dilution refrigerators that provide temperatures of a few millikelvin, it is less easy to incorporate them into a $30 \mathrm{~T}$ hybrid magnet system and the necessarily more restricted space. Particular care has to be taken to avoid eddy current heating associated with time-varying magnetic fields and vibration of conducting parts in non-homogeneous magnetic fields.

We have successfully designed and built a small dilution refrigerator unit which reaches a base temperature of $21 \mathrm{mK}$ at $30 \mathrm{~T}$ and combines the convenience of less-than $24 \mathrm{~h}$ cooldown time with portability. Furthermore, the cryostat insert of overall length $1.8 \mathrm{~m}$ and with a refrigerator unit $70 \mathrm{~cm}$ length inside the vacuum can, fits into the $32 \mathrm{~mm}$ diameter room temperature bore of the Nijmegen $30 \mathrm{~T}$ hybrid magnet system.

In the refrigerator, the circulating ${ }^{3} \mathrm{He}$ gas is condensed on the cold point provided by a pumped ${ }^{4} \mathrm{He}$ pot $(1 \mathrm{~K}$ pot) which takes in liquid helium from the bath. The condenser line passes through the $1 \mathrm{~K}$ pot and contains a flow restriction before it enters the still. The construction of the refrigerator unit is illustrated in Fig. 1. The ${ }^{3} \mathrm{He}$ is led through a continuous heat exchanger and emerges into the mixing chamber at the bottom end of the unit. The heat exchanger consists of a HYSOL rod in which a spiral channel has been machined with a rectangular cross-section of typically $2.5 \times 2.5 \mathrm{~mm}^{2}$. Inside this groove a spiralled capillary carries the flowing concentrated phase. A KAPTON polyimide film is wrapped around and glued onto the outside of the rod, preventing short circuits of the channel. The top section of the capillary is $0.3 \times 0.6 \mathrm{~mm}$ dia. copper-nickel, $1.5 \mathrm{~m}$ length, followed by a section of $1.5 \mathrm{~m} 0.7 \times 1.0 \mathrm{~mm}$ dia. copper-nickel, and a last section of $3 \mathrm{~m} 1.3 \mathrm{~mm}$ inner dia. teflon tubing. The effective exchange surface area is esti-

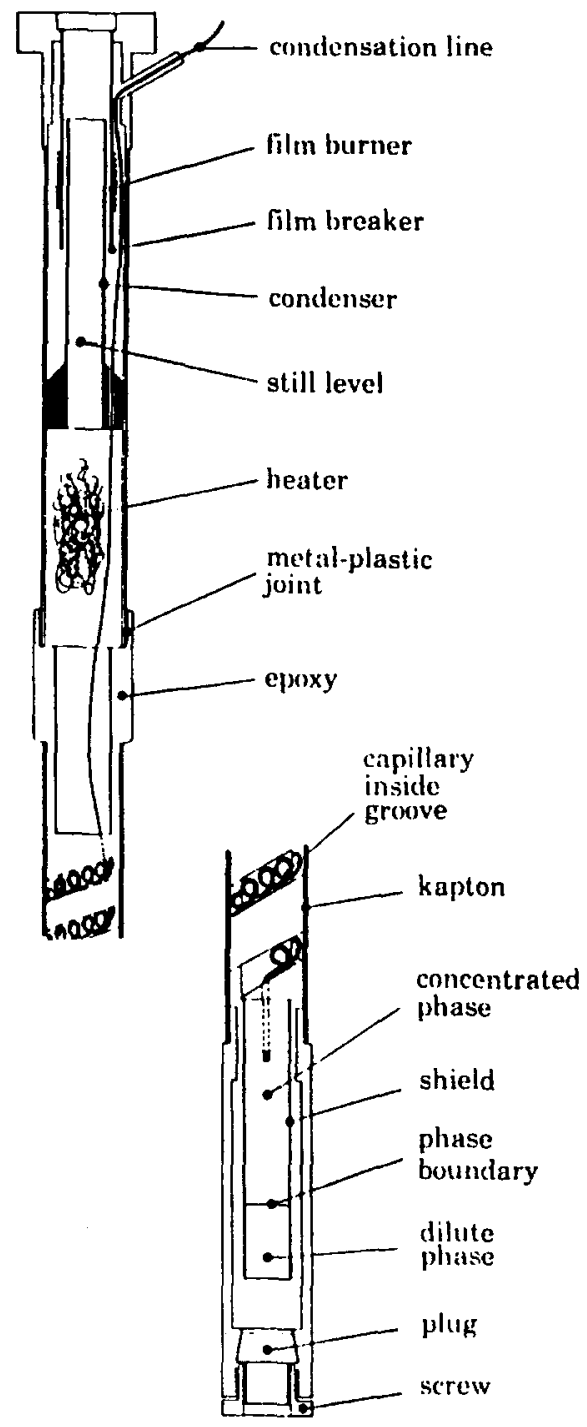

Fig. 1. Schematic representation of the plastic dilution refrigerator insert.

mated to be around $200 \mathrm{~cm}^{2}$. The refrigerator has a ${ }^{3} \mathrm{He}$ charge of $7 \mathrm{dm}^{3}$ NTP.

More recent versions of the lower part of the heat exchanger consist of three rectangular channels located on top of each other and separated by thin KAPTON foil (of 80 and $15 \mu \mathrm{m}$ thickness). The channels are typically $1 \mathrm{~mm}$ deep and several $\mathrm{mm}$ 's wide, and strongly reduce the degradation of the operation due to mutual friction on the dilute side.

The still consists of metal components and between the still and the heat exchanger there is a plastic to metal joint sealed with stycast $2850 \mathrm{GT}$ resin. The still features 


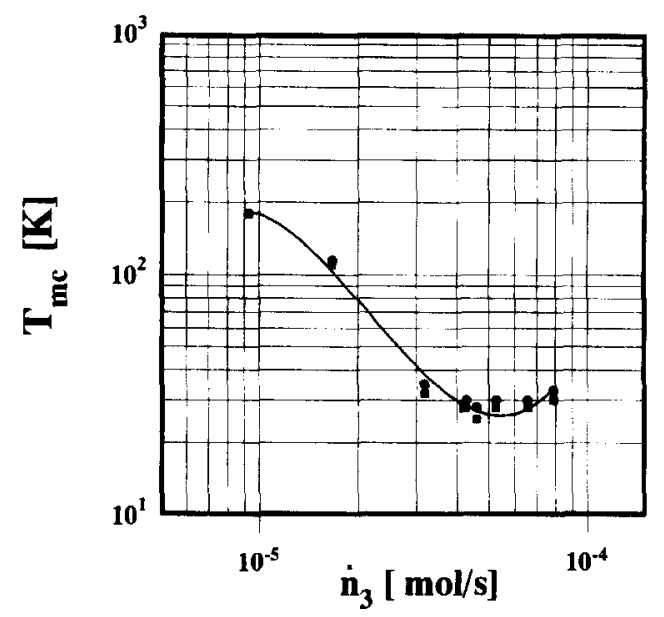

Fig. 2. The base temperature inside the mixing chamber and its dependence on the ${ }^{3} \mathrm{He}$ flow rate for the $\varnothing 32 \mathrm{~mm}$ O.D. unit.

a heater in the liquid and a film burner to reduce the circulation of ${ }^{4} \mathrm{He}$.

The plastic tail is surrounded by the stainless steel tube defining the inner vacuum space and which is immersed in the liquid helium. Spacers are necessary to prevent vibrations and direct touches.

The cylindrical mixing chamber (also machined from HYSOL) contains a concentric shield which forces the concentrated ${ }^{3} \mathrm{He}$ to cross the phase boundary on the inside of the shield. Several thermometers and a heater are mounted, and the wires run up through the groove to the still where they exit the tail via a vacuum seal.

Samples are mounted in the mixture on a leak-tight conical plug at the bottom of the mixing chamber. All wiring necessary for the experiment itself is fed through the plug and runs up on the outside of the heat exchanger and via the still to the copper $1 \mathrm{~K}$ plate.
The base temperature of the mixing chamber with a ${ }^{3} \mathrm{He}$ flow rate of $45 \mu \mathrm{mol} / \mathrm{s}$ is $25 \mathrm{mK}(30 \mathrm{mK}$ at $30 \mathrm{~T}$ ); this can even be brought down to $21 \mathrm{mK}$ when the ${ }^{4} \mathrm{He}$ bath is pumped, due to the reduced background heat leak. The background heat load on the mixing chamber is $1 \mu \mathrm{W}$ and is not only due to thermal conduction from the top through the plastic and the liquid helium, but also through the VESPEL SP22 spacers that touch the $4.2 \mathrm{~K}$ screen. The cooldown time from $4.2 \mathrm{~K}$ to base temperature is about $3 \mathrm{~h}$. Table 2 gives a summary of the most important characteristic values.

The dilution refrigerator has proved itself in several high field experiments $[4,5]$. Some experiments were done with the sample mounted below the mixing chamber in the vacuum: thermal link to the mixture is then made with high purity silver wires fed through the conical plug and connected in the mixing chamber to a mass of sintered silver powder. With this method we have achieved to operate a rotatable sample holder at millikelvin temperatures and in high magnetic fields.

The modular construction of the refrigerator and the fact that there are relatively few parts involved, make it a reliable machine for the experimentalists who have to work with it. It is an advantage that the refrigerator is a $100 \%$ in-house product, so that it is easy to modify and repair.

\section{Acknowledgement}

We are very much indebted to the skill and dedication of J. Rook, in designing and assembling the dilution refrigerator. This project has benefitted from funding by the Commission of the European Communities in its SCIENCE programme.

Table 2

Characteristics of the dilution refrigerator

\begin{tabular}{lll}
\hline & For $32 \mathrm{~mm}$ bore & For $53 \mathrm{~mm}$ bore \\
\hline Sample space & $45 \mathrm{~mm}$ & $45 \mathrm{~mm}$ \\
& $\varnothing 9 \mathrm{~mm}$ & $\varnothing 20 \mathrm{~mm}$ \\
Base temperature $(B=0)$ & $25 \mathrm{mK}$ & $18 \mathrm{mK}$ \\
Base temperature $(B \neq 0)$ & $30 \mathrm{mK} @ 30 \mathrm{~T}$ & $20 \mathrm{mK}^{2} 25 \mathrm{~T}$ \\
Total mixture & $15 \mathrm{dm}^{3} \mathrm{NTP}$ & $40 \mathrm{dm}^{3} \mathrm{NTP}$ \\
${ }^{3} \mathrm{He}$ gas & $7 \mathrm{dm}^{3} \mathrm{NTP}$ & $10 \mathrm{dm}^{3} \mathrm{NTP}$ \\
${ }^{3} \mathrm{He}$ flow rate & $45 \mu \mathrm{mol} / \mathrm{s}$ & $100 \mu \mathrm{mol}_{/} / \mathrm{s}$ \\
Cooling time $4.2 \mathrm{~K} \rightarrow 25 \mathrm{mK}$ & $3 \mathrm{~h}$ & $3 \mathrm{~h}$ \\
\hline
\end{tabular}




\section{References}

[1] J.A.A.J. Perenboom and K. van Hulst, Physica B 155 (1989) 74.

[2] K. van Hulst and J.A.A.J. Perenboom, Physica B 164 (1990) 13.

[3] K. van Hulst and J.A.A.J. Perenboom, IEEE Trans. Magn. 24 (1988) 1397.
[4] S.R. Julian, P.A.A. Teunissen and S.A.J. Wiegers, Phys. Rev. B 46 (1992) 9821.

[5] P.J. Rodgers, C.J.G.M. Langerak, B.L. Gallagher, R.J. Barraclough, M. Henini, G. Hill, S.A.J. Wiegers and J.A.A.J. Perenboom, J. Phys.: Condens. Matter 5 (1993) L449. 